\title{
MBE Growth and Properties of ZnTe- and CdTe-Based Nanowires
}

T. Wojtowicz,* E. Janik, W. Zaleszczyk, J. Sadowski, G. Karczewski, P. DeuŻewski, S. Kret, W. Szuszkiewicz, E. Dynowska, J. Domagala, M. Aleszkiewicz, L. T. Baczewski and A. Petroutchik Institute of Physics, Polish Academy of Sciences, Al. Lotników 32/46, Warszawa 02-668, Poland

\section{A. PRESZ}

Institute of High Pressure Physics, Polish Academy of Sciences, Sokolowska 29/37, Warszawa 01-142, Poland

W. Pacuski, A. Golnik and P. Kossacki

Institute of Experimental Physics, Warsaw University, HoŻa 69, Warszawa 00-681, Poland

\section{J. F. Morhange}

Centre National de la Recherche Scientifique, Université Paris VI et Paris VII, 140 rue de Lourmel, Paris 75015, France

\section{H. Kirmse and W. NeumanN}

Humboldt-Universität zu Berlin, Newtonstrasse 15, D-12489 Berlin, Germany

\section{W. Caliebe}

Hamburger Synchrotronstrahlungslabor at Deutsches Elektronen-Synchrotron, Notkestrasse 85, D-22603 Hamburg, Germany

(Received 10 September 2007)

\begin{abstract}
We review our results on the growth of ZnTe- and CdTe-based nanowires (NWs) and on their basic structural and optical properties. The nanowires were produced by using molecular beam epitaxy (MBE) with the use of a mechanism of catalytically-enhanced growth. The growth of ZnTe, CdTe, $\mathrm{ZnMgTe}$ and ZnMnTe nanowires was performed from elemental $\mathrm{Zn}, \mathrm{Cd}, \mathrm{Mn}, \mathrm{Mg}$ and Te sources on the surfaces of (001)-, (110)- and (111)B-oriented GaAs substrates with Au nanocatalysts. The morphological and structural properties of the nanowires were assessed by using X-ray diffractometry, field-emission scanning electron microscopy, and high resolution transmission electron microscopy. Additional studies of the compositions of both the nanowires and the Au-rich nanocatalysts were performed with the use of energy dispersive X-ray spectroscopy. The optical properties of the NWs were assessed by using photoluminescence and Raman-scattering studies performed in both macro and micro modes. The studies revealed that binary and quaternary nanowires with average diameters from 30 to $70 \mathrm{~nm}$ and lengths from 1 to $2.6 \mu \mathrm{m}$ were monocrystalline in their upper parts, their growth axis was $\langle 111\rangle$, and they grow along the [111] direction of the substrate, independent of the substrate orientation used. A Au-rich (with $20 \% \mathrm{Ga}$ ) spherical nanocatalyst was always visible at the tip of a nanowire, thus indicating that a vapor-liquid-solid mechanism was responsible for the growth of the ZnTe- and the CdTe-based nanowires. The formation of homogeneous mixed crystal ZnMnTe and ZnMgTe nanowires was demonstrated by measurements of the variation of the lattice constant and by Raman experiments that revealed the expected shift and appearance of new phonon lines and a strong enhancement of the LO-phonon structures for an excitation close to the exciton energy of the NW materials. The photoluminescence from the internal $\mathrm{Mn}^{2+}$ transition between crystal-field-split energy levels $\left({ }^{4} \mathrm{~T}_{1} \rightarrow{ }^{6} \mathrm{~A}_{1}\right)$ was observed in the ZnMnTe nanowires.
\end{abstract}

PACS numbers: 68.65.La, 81.15.Hi, 61.72.Cc, 82.65.+r, 61.46.Df

Keywords: Nanowires, Molecular beam epitaxy, Vapor liquid solid, Au catalyst, Diluted magnetic semiconductor, ZnTe, CdTe, ZnMnTe, ZnMgTe, EDX, Raman, Photoluminescence, TEM, SEM

\section{INTRODUCTION}

\footnotetext{
*E-mail: wojto@ifpan.edu.pl; Fax: +48-22-8431331
}

Studies of semiconductor nanowires (NWs) have recently been strongly intensified [1-3] due to the hope of using these 1D structures as "building blocks" for nanoscale electronic and photonic devices [4-6]. Prototype NW-based devices working as light-emitting diodes 
[7] and lasers [2, 8], photodetectors [9], resonant tunnelling diodes [10], field effect [6] and single-electron transistors [11], and biochemical sensors [12] have already been demonstrated.

A broad range of semiconductor nanowires, including Si, Ge [13,14], III-Vs [15,16] and II-VIs [17-21], have been grown using chemical vapor deposition (CVD), thermal evaporation, solvothermal synthesis and molecular-beam exitaxy (MBE) based on the metal-catalyzed vaporliquid-solid (VLS) mechanism [22]. However, only a few reports on the synthesis of tellurium-based NWs have been published so far. ZnTe NWs [20] and CdTe NWs [19] have been obtained by using chemical synthesis or CVD methods. Recently, we have reported the first growth of ZnTe NWs by using the MBE technique [23].

Developing reliable methods of growing $\mathrm{ZnTe}-$ and CdTe-based nanowires and nanowire heterostructures is quite important because such nanostructures play significant roles in the bottom-up approach to nanoelectronics [6] due to the ease of p-type nitrogen and n-type iodine doping of ZnTe and CdTe semiconductors, respectively. Moreover, II-VI tellurides, such as ZnMnTe and CdMnTe, are the best-known diluted magnetic semiconductors (DMS) [24], and carrier-induced ferromagnetism has already been observed in ZnMnTe layers [25] and CdMnTe quantum structures at low temperatures [26]. Therefore II-Te DMS NWs can hopefully be used for the construction of prototype spintronic nanodevices [27] that would take advantage of the spins of carriers in addition to or instead of their charge.

In this paper, we review our results on the growth of ZnTe- and CdTe-based nanowires (NWs) and on their basic structural and optical properties. Specifically, we will demonstrate that high-quality mixedcrystal NWs made of either diluted magnetic semiconductor $\mathrm{Zn}_{1-x} \mathrm{Mn}_{x}$ Te or nonmagnetic semiconductor $\mathrm{Zn}_{1-y} \mathrm{Mg}_{y}$ Te can be produced by using catalytically enhanced MBE.

\section{FABRICATION AND EXPERIMENTS}

The growth of ZnTe- and CdTe-based nanowires was performed in an EPI $620 \mathrm{MBE}$ system equipped with solid-source $\mathrm{Zn}, \mathrm{Cd}, \mathrm{Mg}$ and Te low-temperature effusion cells and a regular cell for $\mathrm{Mn}$. For most of the growth epi-ready (001), (110) and (111)B oriented GaAs substrates were used. Some limited number of growth experiments was done with the use of hybrid substrates made of a 4.5- $\mu$ m-thick CdMgTe epilayer grown on (001) GaAs. Before the growth of the NWs, a 1-nm-thick layer of $\mathrm{Au}$ was deposited in a separate MBE chamber (Ribber $32 \mathrm{R} \& \mathrm{D})$ at $200{ }^{\circ} \mathrm{C}$ without prior removal of the surface oxides. The substrate with a Au layer was then transferred through air to the II-VI MBE growth chamber and in the case of the GaAs substrate was heated in two steps: first to about $580{ }^{\circ} \mathrm{C}$ and then to $550{ }^{\circ} \mathrm{C}$ where it was kept for $10 \mathrm{~min}$. The $\mathrm{Au} / \mathrm{CdMgTe} / \mathrm{GaAs}$ substrate was heated up to $500{ }^{\circ} \mathrm{C}$ and then annealed in a $\mathrm{Cd}$ flux at $450{ }^{\circ} \mathrm{C}$ for $10 \mathrm{~min}$. The MBE growth parameters, such as the substrate temperature, the density of impinging fluxes, and the relative flux ratios were optimized to achieve nanowires that were reproducible from growth to growth, were straight and homogenous in shape, and contained a minimum number of the stacking faults.

The morphology and the structural properties of nanowires were studied by using field-emission scanning electron microscopy (FE-SEM Leo 540), high-resolution transmission electron microscopy (HR-TEM), and X-ray diffractometry (XRD). The samples were prepared for the TEM studies by harvesting nanowires from the original substrate on a holey carbon film supported by a copper grid. TEM investigations were performed either with a JEOL 2200FS system equipped with an incolumn energy filter or with a JEOL 2000EX system. The chemical compositions of both the nanowires and the Au-rich nanocatalysts were investigated by means of energy-dispersive X-ray spectroscopy (EDXS): Röntec system attached to a JEOL 2200FS system. XRD measurements were performed in a symmetrical $\omega-2 \theta$ scan mode by using the characteristic radiation of $\mathrm{Fe}: \mathrm{K}_{\alpha}(\lambda$ $=1.9373 \AA$ ) and $\mathrm{K}_{\beta}(1.75661 \AA)$.

The optical properties of the NWs were assessed by using photoluminescence and Raman scattering studies performed in both the macro and the micro modes. The spectra of either as-grown NWs on GaAs substrate or of NWs removed mechanically from the substrate and deposited onto a Si wafer were collected at temperatures from $8 \mathrm{~K}$ to $295 \mathrm{~K}$ by using $\mathrm{Ar}^{+}$and $\mathrm{Kr}^{+}$laser lines for excitation. During the low-temperature measurements, samples were placed on the cold finger of a continuousflow helium cryostat. Raman scattering measurements were performed in a quasi-backscattering geometry by using a Jobin-Yvon U1000 spectrometer equipped with holographic gratings, a S20 photomultiplier, and a photon counting system. The photoluminescence signal was measured with the use of a CCD camera and a JobinYvon SPEX 270M monochromator. In both the Raman (only at ambient temperature) and the PL studies, spectra of a small number of single NWs could be acquired using the X100 (Raman) or X50 (PL) objective of a microscope coupled to the spectrometer, which ensured a spot diameter of the order of $1-2 \mu \mathrm{m}$.

\section{RESULTS AND DISCUSSION}

\section{ZnTe-based Nanowires}

The structural properties and morphology, the distribution on the substrates and the sizes and orientations of the ZnTe-based nanowires with respect to the substrates, were studied by using scanning and transmis- 

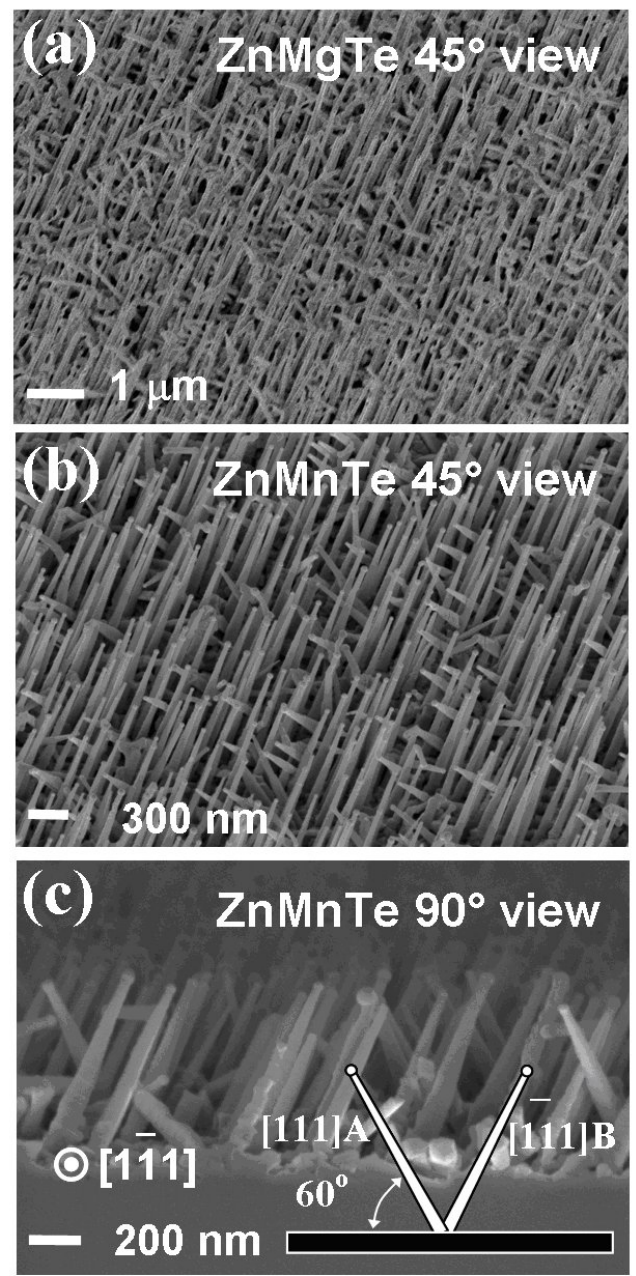

Fig. 1. FE-SEM images of (a) $\mathrm{Zn}_{0.84} \mathrm{Mg}_{0.16} \mathrm{Te}$ and (b) and (c) $\mathrm{Zn}_{0.7} \mathrm{Mn}_{0.3} \mathrm{Te}$ nanowires grown on (110)-oriented GaAs substrates. Panels (a) and (b) represent $45^{\circ}$ views while panel (c) is the side view $\left(90^{\circ}\right)$. The inset in panel (c) show schematic projections onto the plane of the picture of NWs grown along $\langle 111\rangle$ type crystallographic directions of the substrate. The viewing direction and the angles that the projections of the nanowires onto the picture's plane make with the substrate's edge are also given for panel (c).

sion electron microscopy and were used to optimize the growth parameters. We observed that NWs were grown with average diameters (at the top) from 30 to $70 \mathrm{~nm}$ and lengths from 1 to $2.6 \mu \mathrm{m}$, the latter depending on the growth time and flux used. The optimal growth temperature and flux ratio that led to the best-quality NWs was found to a temperature between 360 and $450{ }^{\circ} \mathrm{C}$ and a $\mathrm{Zn} / \mathrm{Te}$ ratio of about 0.65 . At the tip of each $\mathrm{NW}$ a spherical nanocatalyst was always visible (see Figures 1 and 2), thus indicating that a vapor-liquid-solid mechanism was responsible for the growth. Studies of the correlation between the lengths and the diameters of the nanowires, to be discussed in a separate publication, also confirmed an important contribution of the diffusion- induced effect in the VLS growth $[28,29]$. The electron microscopy studies revealed additionally that although the grown nanowires were randomly distributed on the substrates surface, they formed very specific angles both with the substrate normal and with the in-plane substrate directions. The analysis of these angles for NWs grown on (001), (110) and (111)B oriented substrates all lead to the same conclusion. Our ZnTe-based NWs grew practically only along the $\langle 111\rangle$-types of substrate directions, with a preference for $\langle 111\rangle \mathrm{B}$ directions over $\langle 111\rangle$ A directions. Figure 1 shows an example of FESEM studies of mixed-crystal NWs that were grown on a (110)-oriented substrate. In panel (a) we present images of $\mathrm{Zn}_{0.84} \mathrm{Mg}_{0.16} \mathrm{Te} \mathrm{NWs}$, and in panels (b) and (c), we present images of $\mathrm{Zn}_{0.7} \mathrm{Mn}_{0.3}$ Te NWs. The inset in panel (c), which represent the side view, shows schematic projections of NWs grown along $\langle 111\rangle$-type crystallographic directions of the substrate onto the plane of the picture. The viewing direction and the angles which the projections of the nanowires onto the picture's plane make with the substrate's edge are also given for panel (c). By comparing the schematic picture with the real image, NWs are clearly seen to grow along $\langle 111\rangle$ directions of the substrate. Since only two $\langle 111\rangle$ directions stick out of the surface, two families of NWs exist: one along the $\langle 111\rangle \mathrm{A}$ type direction and the other along the $\langle 111\rangle \mathrm{B}$ type direction. In the case of (100)-oriented substrates, two types of $\langle 111\rangle \mathrm{A}$ NWs and two types of $\langle 111\rangle \mathrm{B}$ NWs were observed, all at the angle of $54.7^{\circ}$ to the substrate normal, as expected. Finally, for the (111)B-oriented substrates three types of $\langle 111\rangle \mathrm{A} \mathrm{NWs}$, making an angle of $70.5^{\circ}$ with the substrate normal, and one types of $\langle 111\rangle \mathrm{B}$ NWs, perpendicular to the substrate surface, were seen.

The SEM studies strongly suggest that the $\langle 111\rangle$ direction is the direction of the growth axis of all our ZnTe-based nanowires. This was confirmed by using $\mathrm{X}$-ray diffractometry, to be discussed later, and by using TEM studies, such as those exemplified in Figure 2. The measurements of the relative interplanar distances and angles on the HR-TEM images and the selected area electron diffraction (SAED) patterns proved that ZnTebased NWs had a zinc-blende (ZB) structure and that their growth axis was along the $\langle 111\rangle$ direction. The HR-TEM studies also showed that NWs grown at correct conditions were defect free in their upper parts, although in most cases they did posses stacking faults and twins in their lower parts. As an example, the upper part of Figure 2 presents a TEM image of the ZnTe nanowires that were grown on a (110)-oriented GaAs substrate and were then transferred to a holey carbon film supported by a copper grid for the TEM studies. In this image, the $\mathrm{Au}$-based nanocatalyst particles are even more clearly visible then in SEM images presented in Figure 1. In panel (b), an image of the expanded region of the lower part of one of the NWs is shown, and in panel (c), the corresponding SAED is shown. These data reveal the ZB structure and the $\langle 111\rangle$ growth axis of the NW and the 


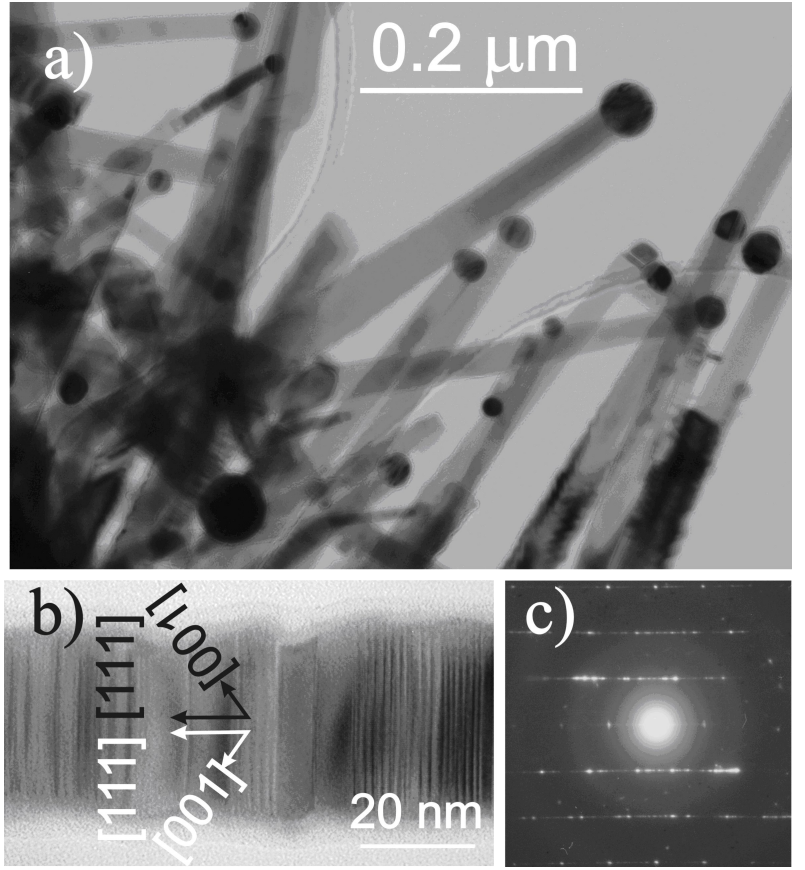

Fig. 2. (a) TEM image of ZnTe nanowires removed from the original (110)-oriented GaAs substrate on which they were grown and deposited on a perforated carbon film. Aubased catalyst particles are visible at the tip of each NW (b) - an image of the expanded region of the lower part of one of the NWs and (c) corresponding SAED.

presence of twins.

The TEM and SEM results discussed above indicate that NWs grow on GaAs substrates epitaxially, a conclusion that is also supported by X-ray diffraction studies, to be discussed later, but the question arises how the epitaxy of the NWs in directions other than the substrate normal is at all possible. Krishnamachari et al. [30] proposed that during the formation of nanocatalyst particles, the GaAs substrate is locally dissolved in a reaction with the Au layer and that low-energy $\{111\}$ facets develop within the pit. Such facets can be starting points for the epitaxial growth of the $\langle 111\rangle$-oriented NWs observed in our experiments for all substrate orientations. This scenario in our growth processes is confirmed by the results of EDX studies of the chemical compositions of nanoparticles present at the tips of all our NWs, showing that these nanoparticles are solidified eutectic alloys of $\mathrm{Au}$ and $\mathrm{Ga}$, with a small addition of $\mathrm{Zn}$. The results of a semi-quantitative analysis showed: 75 at.\% of $\mathrm{Au}$, about 22 at.\% of $\mathrm{Ga}$ and about 3 at.\% of $\mathrm{Zn}$.

Energy dispersive X-ray spectroscopy technique was also used to study the chemical compositions of the nanowires themselves. These studies were particularly useful in proving that Mn atoms were incorporated into the growing ZnTe NWs to form mixed crystal NWs. An example of such studies is presented in Figure 3. In the left panel, the Z-contrast scanning-TEM image of a single $\mathrm{Zn}_{1-x} \mathrm{Mn}_{x}$ Te nanowire that was grown on (001)-
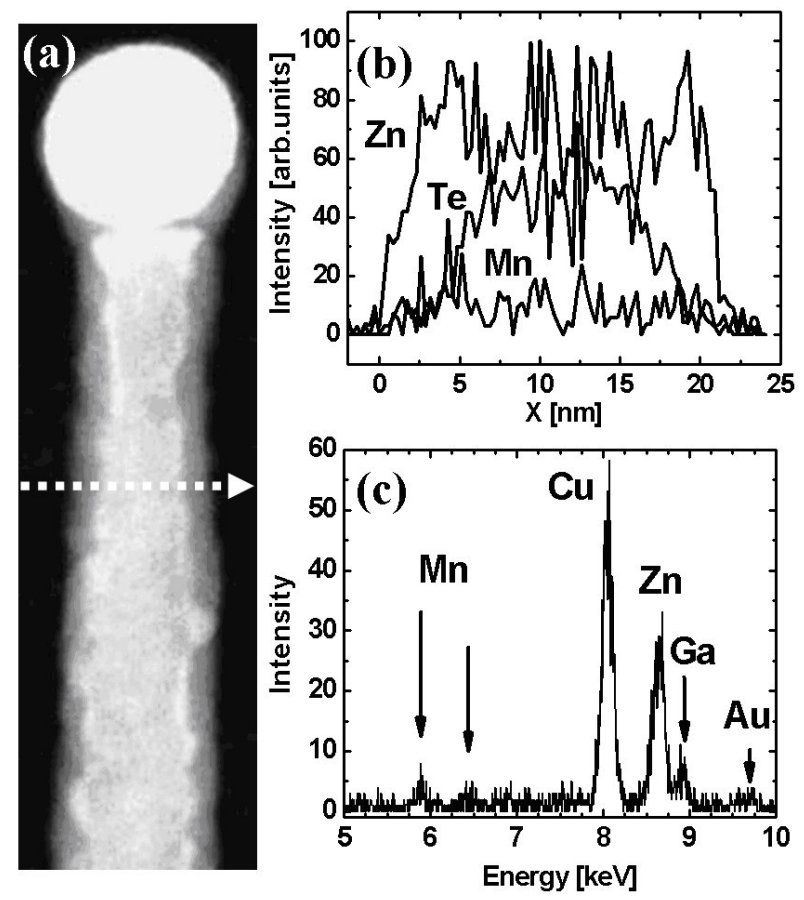

Fig. 3. (a) Z-contrast scanning-TEM and (b) EDX analysis of the single $\mathrm{Zn}_{1-x} \mathrm{Mn}_{x}$ Te nanowire. Panel (b) shows the results of the EDX scan along the dashed line in panel (a) perpendicular to the growth axis of the NW. In panel (c), one of the collected point EDX spectra is presented.

oriented GaAs is presented. Panel (b) shows the results of the EDX scan along the dashed line perpendicular to the growth axis of the NW presented in panel (a). In panel (c), one of the collected EDX spectra is presented. Although the characteristic Mn peaks in panel (c) have small amplitudes, leading to quite substantial scattering of the points in the Mn line scan in panel (b), the presence on $\mathrm{Mn}$ is clearly revealed.

The formation of the $\mathrm{Zn}_{1-x} \mathrm{Mn}_{x}$ Te and $\mathrm{Zn}_{1-y} \mathrm{Mg}_{y} \mathrm{Te}$ mixed-crystal NWs, as well as the existence of a preferred orientation of the NWs with respect to the substrate, was demonstrated by using X-ray diffractometry. Figure 4 presents examples of the diffraction patterns obtained around 004 reflections in symmetrical $\omega-2 \theta$ scans for $\mathrm{ZnTe}, \mathrm{Zn}_{1-x} \mathrm{Mn}_{x} \mathrm{Te}$, and $\mathrm{Zn}_{1-y} \mathrm{Mg}_{y}$ Te nanowires grown on (001)-oriented GaAs substrates. One can clearly see a shift of both the $K_{\alpha}$ and the $K_{\beta} 004$ reflections (marked by arrows) of the mixed-crystal nanowires towards smaller angles with respect to that of pure ZnTe. This shift can be used to determine the change in the lattice constant of the nanowire material and, by assuming a dependence of the lattice constant on the composition known for the bulk quaternary materials, allows the compositions of $\mathrm{Mg}$ and $\mathrm{Mn}$ (the values obtained for the samples presented in Figure 3 are also given) to be determined. For the $\mathrm{Zn}_{1-x} \mathrm{Mn}_{x}$ Te and the $\mathrm{Zn}_{1-y} \mathrm{Mg}_{y}$ Te NWs grown so far, the maximum compositions determined by using this method were found to be $x=0.3$ and $y=0.16$, 


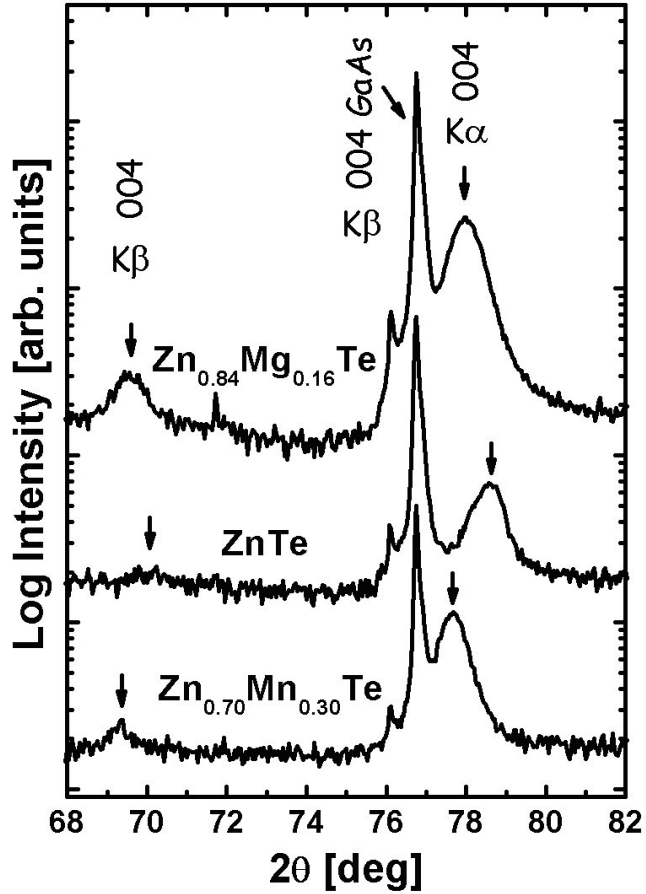

Fig. 4. X-ray diffraction patterns obtained around 004 reflections in symmetrical $\omega-2 \theta$ scans for $\mathrm{ZnTe}, \mathrm{Zn}_{1-x} \mathrm{Mn}_{x} \mathrm{Te}$, and $\mathrm{Zn}_{1-y} \mathrm{Mg}_{y}$ Te nanowires grown on (001)-oriented GaAs substrates. The peaks from the NWs are marked by arrows. Values of $\mathrm{x}$ and $\mathrm{y}$, given in the figure, were determined from the measured lattice constant change.

respectively.

Apart from the strong 002, 004 and 006 reflections from the NWs, in the data collected in the wider range of $2 \theta$ angles by using synchrotron radiation at the W1.1 beamline at Deutsches Elektronen-Synchrotron - Hamburger Synchrotronstrahlungslabor (not shown), additional small peaks (e.g., indexed 111, 220, 311) were seen. These peaks were interpreted as being related to a thin polycrystalline layer (also visible in Figure 1(c)) based on the experiments performed in the grazing incidence geometry. The fact that in symmetrical scans, the strongest among the observed reflections of ZnTebased nanowires are indexed 002, 004 and 006 indicates that the (001) lattice planes in nanowires are parallel to the (001) lattice planes in the substrate. As a consequence, these nanowires must be inclined at an angle of $54.7^{\circ}$ toward the [001] crystallographic direction of the substrate because they grow along $\langle 111\rangle$ crystallographic directions (the angle between the crystallographic directions $\langle 111\rangle$ and [001] in a cubic system is equal to $\left.54.7^{\circ}\right)$. The X-ray studies of other nanowires grown on (110)and (111)-oriented GaAs lead to similar conclusions: in the first case, the nanowires are inclined toward the direction of the substrate at an angle of $35.3^{\circ}$ because (110) planes are parallel to (110) planes of the substrate while the nanowires grown on (111) oriented substrate are either perpendicular to the substrate surface or form a

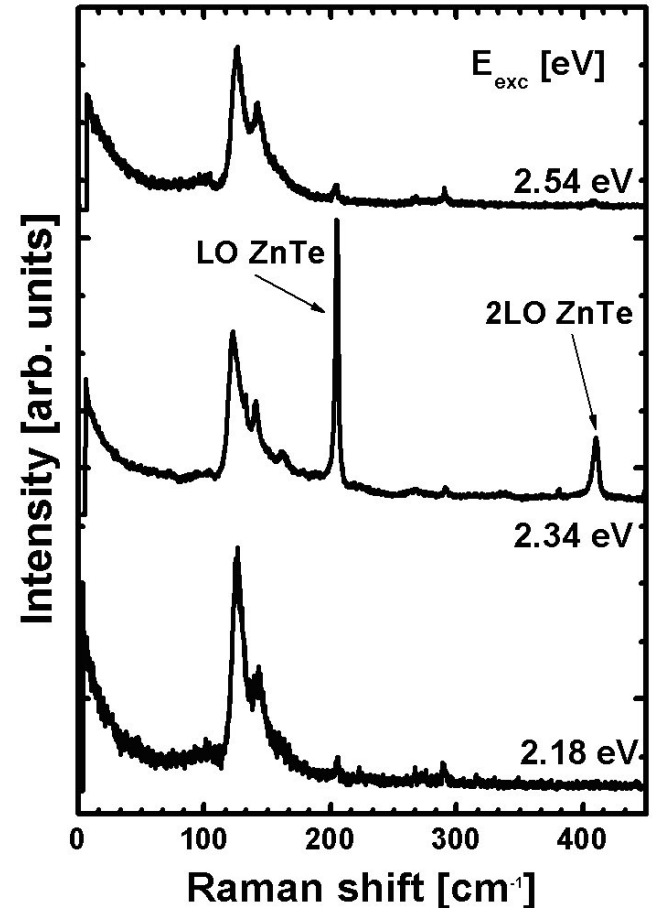

Fig. 5. Raman scattering spectra of as-grown ZnTe/GaAs(001) NWs excited at room temperature with laser lines of various energies: 2.54, 2.34 and $2.18 \mathrm{eV}$.

$70.5^{\circ}$ angle with the substrate normal. Therefore, X-ray diffractometry constitutes strong support of the epitaxial relation between our ZnTe-based NWs and GaAs substrates, observed in FE-SEM studies, and gives evidence for the formation of mixed-crystal nanowires.

More strong evidence for the formation of the $\mathrm{Zn}_{1-x} \mathrm{Mn}_{x} \mathrm{Te}$ and $\mathrm{Zn}_{1-y} \mathrm{Mg}_{y} \mathrm{Te}$ mixed-crystal nanowires and the high quality of our ZnTe-based NWs is provided by the optical studies. Figure 5 presents the Raman scattering spectra of as-grown ZnTe NWs that were grown on (001)-oriented GaAs substrate. The spectra were collected at room temperature by using various laser lines with photon energies of $2.54,2.34$, and $2.18 \mathrm{eV}$. It is important to underline that the spectra collected from NWs removed from the original substrate and deposited on $\mathrm{Si}$ (e.g. shown in Figure 6 for the $\mathrm{Zn}_{1-y} \mathrm{Mg}_{y} \mathrm{Te}$ NWs) are very similar and differ only by the presence of the strong Si Raman peak. Therefore, the ZnTe-based phonon features visible in Figure 5 are NWs related and are not caused by the Raman scattering in the polycrystalline layer that forms on the GaAs substrate during the growth of NWs. In Figure 5, the line near $209 \mathrm{~cm}^{-1}$ coincides with the LO phonon line observed in bulk crystals of ZnTe. Also clearly visible in this figure is a strong enhancement of the intensity of this line and the appearance of an additional line at double the frequency of the ZnTe LO-phonon in spectra collected when using a laser having a photon energy equal to $2.34 \mathrm{eV}$, a value which is very close to the band gap energy of ZnTe at room tem- 


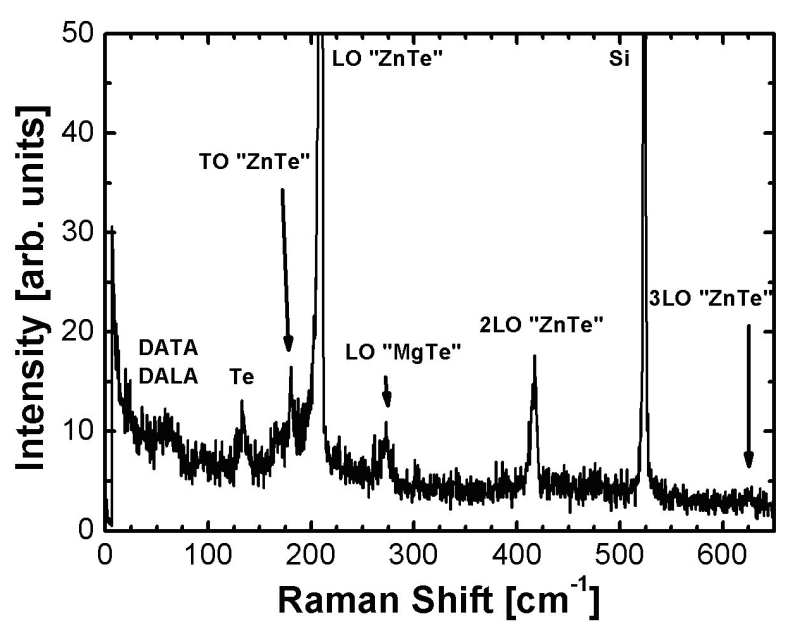

Fig. 6. Raman scattering spectrum of $\mathrm{Zn}_{0.84} \mathrm{Mg}_{0.16} \mathrm{Te}$ NWs removed from a (110)-GaAs substrate and deposited onto a Si wafer. Data were collected with the use of a 514.5$\mathrm{nm}$ laser line at $15 \mathrm{~K}$.

perature. The observation of well-defined, narrow LOphonon structures and its replicas (up to the 6-th at low temperatures, not shown here) at the resonant conditions is direct proof of the good crystalline quality of the grown NWs. Apart from the ZnTe LO phonon lines some additional features were observed near 127 and $143 \mathrm{~cm}^{-1}$ and were attributed to the optical phonons of hexagonal tellurium. Precipitates of this material are usually present in MBE-grown tellurium compounds and are easily observed by using Raman spectroscopy because of the high cross section of this material.

As an example of the Raman studies of mixed-crystal NWs, in Figure 6, we present the spectra collected from $\mathrm{Zn}_{1-y} \mathrm{Mg}_{y} \mathrm{Te}$ nanowires grown on (110)-oriented GaAs. The experiments were performed at $15 \mathrm{~K}$ with a 514.5 $\mathrm{nm}$ laser line on nanowires that were transferred onto a Si substrate (hence, the Raman peak from Si is visible in the spectra). It is well known from Raman scattering measurements on the bulk crystals [31] that, due to the significant mass difference between $\mathrm{Zn}$ and $\mathrm{Te}$ on the one hand and $\mathrm{Mg}$ on the other, the $\mathrm{Zn}_{1-y} \mathrm{Mg}_{y}$ Te alloys exhibit a "two-mode" behavior. In fact, as expected for two-mode mixed-crystal NWs, the spectrum in Figure 6 consists of shifted to smaller wavenumber "ZnTe-like" TO and LO lines (labeled in the figure as TO "ZnTe" and LO "ZnTe", respectively) and "MgTe-like" LO line (labeled as LO "MgTe"). Also, as in the case of Raman studies of ZnTe NWs described before, in the present spectra the LO replicas (2LO and $3 \mathrm{LO}$ ) of "ZnTe-like" modes are observed, proving the good crystalline quality of the grown NWs. The concentration of $\mathrm{Mg}$ in the NWs estimated from the Raman scattering by comparison of the line spectral positions of the lines with those of bulk $\mathrm{Zn}_{1-y} \mathrm{Mg}_{y}$ Te crystals [31], corresponds quite well to that resulting from the $\mathrm{X}$-ray diffraction measurements.

Similar experiments performed on $\mathrm{Zn}_{1-x} \mathrm{Mn}_{x}$ Te NWs

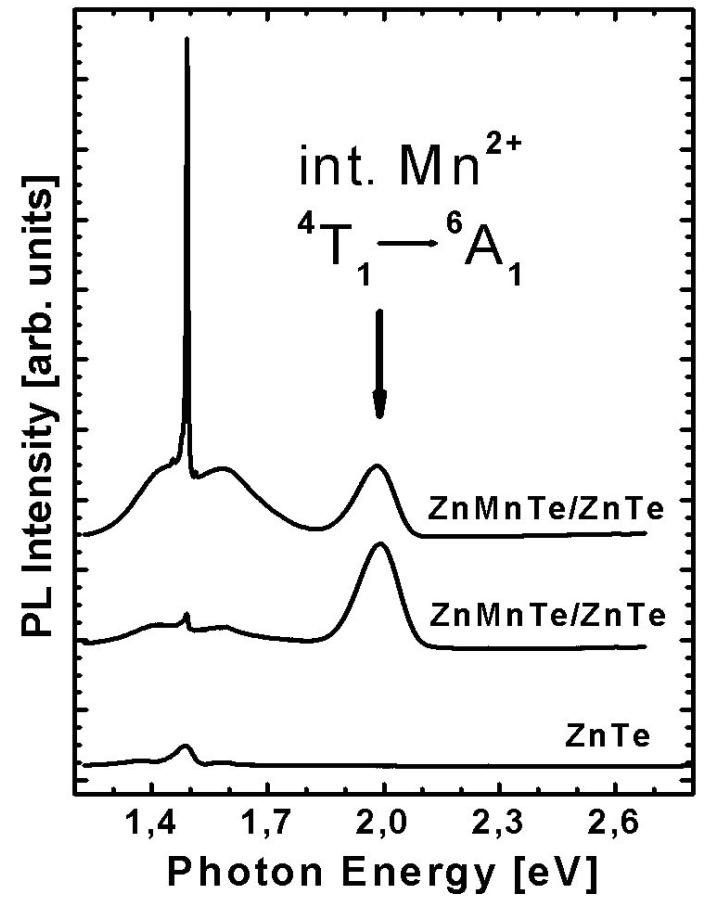

Fig. 7. Photoluminescence of the two $\mathrm{Zn}_{1-x} \mathrm{Mn}_{x}$ Te and one ZnTe NW samples. Spectra were excited for the as-grown samples by a $390-\mathrm{nm}$ laser line at $8 \mathrm{~K}$.

also revealed spectra with shifts and splittings of Raman lines comparable to those observed in bulk $\mathrm{Zn}_{1-x} \mathrm{Mn}_{x} \mathrm{Te}$ mixed crystal [32] with a composition equal to the composition of NWs estimated based on their lattice constant. In the case of $\mathrm{Zn}_{1-x} \mathrm{Mn}_{x}$ Te NWs, additional support for the substitutional incorporation of Mn into the zinc site of the lattice comes from the observation of the strong photoluminescence line centered at an energy around $2 \mathrm{eV}$. The line is visible, for instance, in PL spectra of two samples containing $\mathrm{Zn}_{1-x} \mathrm{Mn}_{x}$ Te NWs, presented in Figure 7, and absent in ZnTe NW sample. This $\mathrm{PL}$ line is characteristic of the internal $\mathrm{Mn}^{2+}$ transition between energy levels $\left({ }^{4} \mathrm{~T}_{1} \rightarrow{ }^{6} \mathrm{~A}_{1}\right)$ split by the tetrahedral crystal field [33].

\section{CdTe-based Nanowires}

Two types of the CdTe-based structures were grown and studied: homogenous CdTe nanowires (grown either on (001)-oriented GaAs substrates or on hybrid CdMgTe/GaAs-(001) substrates) and CdTe NWs grown on ZnTe NW pedestals. In Figure 8, we present an example of the TEM studies of a single CdTe NW grown by using atomic layer epitaxy on the (001)-oriented Cd$\mathrm{MgTe} / \mathrm{GaAs}$ hybrid substrate. During the growth process, $700 \mathrm{Cd}$ and Te flux cycles, each with a duration of $5 \mathrm{~s}$ (for the $\mathrm{Cd}$ and the Te fluxes used, each pulse corresponded to the supply of material for the growth of 


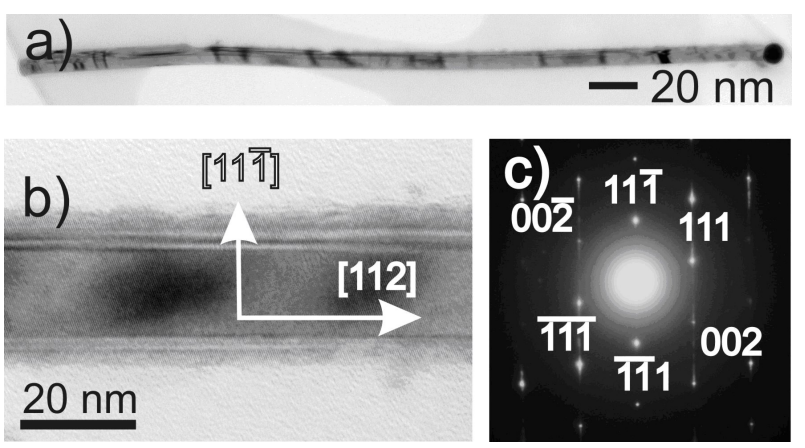

Fig. 8. (a) TEM image of a single CdTe NW grown on a (001)-oriented CdMgTe/GaAs hybrid substrate by using atomic layer epitaxy. An (b) image and a (c) diffraction pattern from a fragment of the same CdTe NW, which demonstrates the [112] growth direction of the NW.

3 CdTe MLs at normal growth temperature), were supplied, which would have led to at most a $0.1-\mu$ m-thick layer (assuming 0.5 ML per cycle) if not for the $\mathrm{Au}-$ catalyst VLS growth mode. The nanowires total length was above $1.8 \mu \mathrm{m}$; therefore, an enhancement of the linear growth rate by a factor of about 20 was observed. In panels (a) and (b) of Figure 8, the TEM images acquired with increasing magnification are shown. In panel (b) the interplanar, distances correspond to that of zinc-blende CdTe. In panel (c), the selected area electron diffraction pattern from the fragment presented in panel (b) is shown, which demonstrates the [112] growth direction of the NW. This indicates that in the case when no GaAs substrate decomposition occurs (since Au layer was deposited on a thick CdMgTe buffer layer, and a lower temperature of $450{ }^{\circ} \mathrm{C}$ was used for nanocatalyst formation), the growth of CdTe nanowires having a growth axis other than the $\langle 111\rangle$ directions, such as the $\langle 112\rangle$ direction in this case, can be achieved. This result is important because it demonstrates that the growth direction of II-Te NWs by catalytic MBE can be different than the typical $\langle 111\rangle$ direction.

For the future possible applications of II-Te NW structures as single-photon sources on demand, it would be necessary to master the growth of a single CdTe quantum dot embedded into a NW made of a material having an energy gap larger that that of CdTe. Therefore, a part of our research was devoted to the growth of a CdTe $\left(E_{g}=1.6 \mathrm{eV}\right)$ segment inside a ZnTe $\left(E_{g}=2.4\right.$ eV) NW. Figure 9 presents the results of TEM studies of a single CdTe NW grown on the ZnTe NW pedestal. The growth procedure was the following. First, $0.8 \mu \mathrm{m}$ long ZnTe NWs were grown on (110)-GaAs with the use of a previously established procedure [23]. Then, the Zn flux was stopped, and the Cd flux was supplied. As one can see in panel (a), this extended the ZnTe NWs by initially thicker, additional segments made of CdTe.

In order to evaluate the intermixing of the interface between ZnTe and CdTe, we performed a geometric phase analysis GPA [34] of the TEM 002 lattice fringes of the

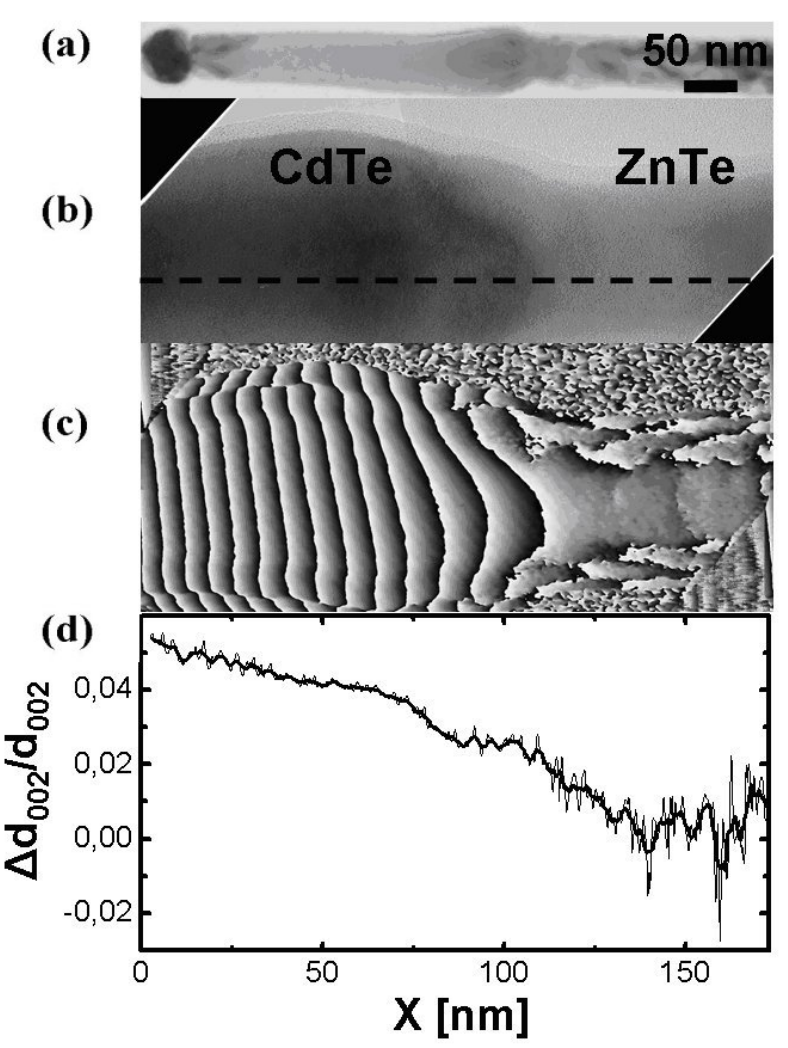

Fig. 9. (a) and (b) TEM images and geometric phase analysis of a single CdTe NW grown on a $\langle 111\rangle$-oriented ZnTe NW pedestal. The ZnTe NWs were grown on a (110)-oriented GaAs substrate. Panel (c) shows the geometric phase, $\mathrm{P}_{002}$, for the 002 lattice fringes of the expanded region of NW heterostructures in the vicinity of the CdTe/ZnTe interface. The relative change of the 002 interplanar distance along the dashed line in panel (b) is presented in panel (d)

transitional NW region, the TEM of which is presented in panel (b) of Figure 9. A visualization of the geometric phase P002 (shift between the 002 lattice finger's position and the reference lattice fringes with the constant period calculated as the average value for the ZnTe part of the NW) is shown in panel (c) of Figure 9. The gradient of such a calculated geometric phase is stronger when the period of the fringes becomes closer to those of CdTe. As we can see, the gradient of the phase $\mathrm{P}_{002}$ is not abrupt, but changes progressively, what indicates quite substantial intermixing of $\mathrm{ZnTe}$ and $\mathrm{CdTe}$, both along and perpendicular to the NW axis.

With the GPA, the profiles of the relative changes of the lattice constant $\Delta d_{002} / d_{002}$ can be obtained. This can be interpreted, in first the approximation, as the composition profile in the growth direction (a precise determination of the composition by using the strain measurement needs an extended simulation of the surface relaxation phenomena and projection/averaging along ebeam). Such a profile along the dashed line in panel (b) of Figure 9 is presented in panel (d). At a distance of 130 $\mathrm{nm}$, a relative change in the lattice constant of about 0.05 
was observed, with the total $\Delta d_{002} / d_{002}$ between CdTe and ZnTe being 0.062. Therefore, the total length of the transitional region from ZnTe to CdTe is of the order of $150 \mathrm{~nm}$. These data indicate that the $\mathrm{Cd}$ adatoms, while moving on the side surface of the previously grown ZnTe pedestal, diffuse into the ZnTe crystal lattice and that the intermixed region (made of $\mathrm{CdZnTe)} \mathrm{extends}$ over a distance nearly three times larger than the diameter of the eutectic ball. The effect of this intermixing can hopefully be reduced by decreasing the CdTe growth temperature (so far this temperature was the same as for the growth of the ZnTe pedestal), by using growth interruption in the Te flux at the boundary between ZnTe and CdTe, and by improving the stoichiometry of the ZnTe pedestal, because diffusion is known to precede mainly through metal vacancies [35].

\section{SUMMARY}

In conclusion we have reviewed our results on the growth of ZnTe- and CdTe-based nanowires and on their basic structural and optical properties. The nanowires were grown by using catalytically enhanced molecular beam epitaxy (MBE) based on Au nanocatalysts. With the use of various experimental technique: FE-SEM, TEM, X-ray diffractometry, EDXS, PL, and Raman scattering, we demonstrated that not only high-quality binary ZnTe and CdTe nanowires but also nanowires made of mixed $\mathrm{Zn}_{1-x} \mathrm{Mn}_{x}$ Te and $\mathrm{Zn}_{1-y} \mathrm{Mg}_{y}$ Te crystals could be produced by using MBE. Additionally the first growth of $\mathrm{CdTe} / \mathrm{ZnTe} \mathrm{NW}$ heterostructures was reported. Our studies revealed that binary and quaternary nanowires with average diameters from 30 to $70 \mathrm{~nm}$ and lengths from 1 to $2.6 \mu \mathrm{m}$ were monocrystalline in their upper parts, their growth axis were along the $\langle 111\rangle$ direction, and they grow along the $\langle 111\rangle$ direction of the substrate, independent of the orientation of the substrate used. We believe that our results are important because NWs based on ZnTe and CdTe can play particularly important roles in the bottom-up approach to nanoelectronics due to the ease of p-type nitrogen and n-type iodine doping of these semiconductors, respectively. Additionally the successful growth of $\mathrm{Zn}_{1-x} \mathrm{Mn}_{x}$ Te nanowires by MBE opens new perspectives for applications in prototype spintronic nanodevices that would take advantage of the spins of carriers in addition to or instead of their charge [27].

\section{ACKNOWLEDGMENTS}

This research was partially supported by the Ministry of Science and Higher Education (Poland) through Grants N507 030 31/0735 and N515 015 32/0997, by the Network "New Materials and Sensors for Optoelectronics, Information technology, Energetic applications, and Medicine", and by the Foundation for Polish Science through Subsidy 12/2007.

\section{REFERENCES}

[1] L. Samuelson et al., Phys. E 25, 313 (2004).

[2] M. S. Gudiksen, L. J. Lauhon, J. Wang, D. C. Smith and C. M. Lieber, Nature 415, 617 (2002).

[3] W. Seifert et al., J. Cryst. Growth 272, 211 (2004).

[4] Y. Huang, X. Duan, Y. Cui, L. J. Lauhon, K. H. Kim and C. M. Lieber, Science 294, 1313 (2001).

[5] L. Samuelson, Materials Today 6, 22 (2003).

[6] Y. Cui and C. M. Lieber, Science 291, 851 (2001).

[7] F. Qian, Y. Li, S. Gradecak, D. Wang, C. J. Barrelet and C. M. Lieber, Nano Lett. 4, 1975 (2004).

[8] X. Duan, Y. Huang, R. Agarwal and C. M. Lieber, Nature 421, 241 (2003).

[9] J. Wang, M. S. Gudiksen, X. Duan, Y. Cui and C. M. Lieber, Science 293, 1455 (2001).

[10] M. T. Björk, B. J. Ohlsson, C. Thelander, A. I. Persson, K. Deppert, L. R. Wallenberg and L. Samuelson, Appl. Phys. Lett. 81, 4458 (2002).

[11] C. Thelander, T. Martensson, M. T. Björk, B. J. Ohlsson, M. W. Larsson, L. R. Wallenberg and L. Samuelson, Appl. Phys. Lett. 83, 2052 (2003).

[12] Y. Cui, Q. Wei, H. Park and C. M. Lieber, Science 293, 1289 (2001).

[13] S. Kodambaka, J. Tersoff, M. C. Reuter and F. M. Ross, Phys. Rev. Lett. 96, 096105 (2006).

[14] P. Werner, N. D. Zakharov, G. Gerth, L. Schubert, L. Sokolov and U. Gösele, J. Cryst. Growth 290, 6 (2006).

[15] B. J. Ohlsson, M. T. Björk, M. H. Magnusson, K. Deppert, L. Samuelson and L. R. Wallenberg, Appl. Phys. Lett. 79, 3335 (2001)

[16] X. Duan, Y. Huang, Y. Cui, J. Wang and C. M. Lieber, Nature 409, 66 (2001).

[17] S. Kar, S. Biswas and S. Chaudhuri, Nanotechnology 16 , $737(2005)$.

[18] A. Colli, S. Hofmann, A. C. Ferrari, F. Martelli, S. Rubini, C. Ducati, A. Franciosi and J. Robertson, Nanotechnology 16, S139 (2005).

[19] R. Krahne, G. Chilla, C. Schuller, L. Carbone, S. Kudera, G. Mannarini, L. Manna, D. Heitmann and R. Cingolani, Nano Lett. 6, 478 (2006).

[20] H. B. Huo, L. Dai, C. Liu, L. P. You, W. Q. Yang, R. M. Ma, G. Z. Ran and G. G. Qin, Nanotechnology 17, $5912(2006)$.

[21] S. K. Chan, Y. Cai, N. Wang and I. K. Sou, J. Cryst. Growth 301-302, 866 (2007).

[22] R. S. Wagner and W. C. Ellis, Appl. Phys. Lett. 4, 89 (1964).

[23] E. Janik et al., Appl. Phys. Lett. 89, 133114 (2006).

[24] J. K. Furdyna, J. Appl. Phys. 64, R29 (1988).

[25] D. Ferrand et al., Phys. Rev. B 63, 085201 (2001).

[26] P. Kossacki, A. Kudelski, J. A. Gaj, J. Cibert, S. Tatarenko, D. Ferrand, A. Wasiela, B. Deveaud and T. Dietl, Phys. E 12, 344 (2002).

[27] S. A. Wolf, D. D. Awschalom, R. A. Buhrman, J. M. Daughton, S. von Molnar, M. L. Roukes, A. Y. Chtchelkanova and D. M. Treger, Science 294, 1488 (2001). 
[28] V. G. Dubrovskii, G. E. Cirlin, I. P. Soshnikov, A. A. Tonkikh, N. V. Sibirev, Y. Samsonenko and V. M. Ustinov, Phys. Rev. B 71, 205325 (2005).

[29] V. G. Dubrovskii, N. V. Sibirev, G. E. Cirlin, J. C. Harmand and V. M. Ustinov, Phys. Rev. E 73, 021603 (2006).

[30] U. Krishnamachari, M. Borgstrom, B. J. Ohlsson, N. Panev, L. Samuelson, W. Seifert, M. W. Larsson and L. R. Wallenberg, Appl. Phys. Lett. 85, 2077 (2004).

[31] R. Vogelgesang, A. J. Mayur, M. D. Sciacca, E. Oh,
I. Miotkowski, A. K. Ramdas, S. Rodriguez and G. Bauer, J. Raman Spectrosc. 27, 239 (1996).

[32] D. L. Peterson, A. Petrou, W. Giriat, A. K. Ramdas and S. Rodriguez, Phys. Rev. B 33, 1160 (1986).

[33] E. Müller and W. Gebhardt, Phys. Stat. Sol. B 137, 259 (1986).

[34] M. J. Hytch, E. Snoeck and R. Kilaas, Ultramicroscopy 74, 131 (1998).

[35] A. Barcz, G. Karczewski, T. Wojtowicz, M. Sadlo and J. Kossut, Appl. Phys. Lett. 72, 206 (1998). 